TITLE:

\title{
A phosphodiesterase III inhibitor protects rat liver from sinusoidal obstruction syndrome through heme oxygenase- 1 induction
}

\section{$\operatorname{AUTHOR}(\mathrm{S})$ :}

Narita, Masato; Hatano, Etsuro; Ikai, Iwao; MiyagawaHayashino, Aya; Yanagida, Atsuko; Nagata, Hiromitsu; Asechi, Hiroyuki; Taura, Kojiro; Uemoto, Shinji

\section{CITATION:}

Narita, Masato ... [et al]. A phosphodiesterase III inhibitor protects rat liver from sinusoidal obstruction syndrome through heme oxygenase-1 induction. Annals of Surgery 2009, 249(5): 806-813

\section{ISSUE DATE:}

2009-05

URL:

http://hdl.handle.net/2433/84840

\section{RIGHT:}

c 2009 by Lippincott Williams \& Wilkins. 許諾条件により本文は2010-0601に公開.; この論文は出版社版でありません。引用の際には出版社版 をご確認ご利用ください。; This is not the published version. Please cite only the published version. 


\section{A phosphodiesterase III inhibitor protects rat liver from sinusoidal obstruction}

\section{syndrome through heme oxygenase-1 induction}

Masato Narita, MD, ${ }^{*}$ Etsuro Hatano, MD, PhD,* Iwao Ikai, MD, PhD,* Aya Miyagawa-Hayashino, MD,† Atsuko Yanagida, MD,* Hiromitsu Nagata, MD,* Hiroyuki Asechi, MD,* Kojiro Taura, MD, PhD*, Shinji Uemoto, MD, PhD*

* Department of Surgery, Graduate School of Medicine, Kyoto University, Kyoto, Japan $\dagger$ Department of Diagnostic Pathology, Kyoto University Hospital, Kyoto, Japan

\section{Correspondence to: Hatano, E}

Department of Surgery, Graduate school of Medicine, Kyoto University

54 Kawahara-cho, Syogoin, Sakyo-ku, Kyoto 606-8507, Japan

Telephone number: 81-75-751-3234, Fax number: 81-75-751-4263

E-mail: etsu@kuhp.kyoto-u.ac.jp

This study was supported in part by grants from the Scientific Research Fund of the Ministry of Education of Japan and from the Public Trust Surgery Research Fund.

Running head: Olprinone protects rat liver from SOS 


\section{Mini-Abstract}

Sinusoidal obstruction syndrome (SOS) induced by chemotherapy is of concern in hepatectomy for patients with hepatic colorectal metastases. We succeeded in inhibiting the occurrence of SOS in a rat model through induction of heme oxygenase-1 by pretreatment with olprinone, a phosphodiesterase III inhibitor. 


\section{Abstract}

Objective: The aim of study was to investigate pharmacological treatment for sinusoidal obstruction syndrome (SOS).

Background: SOS is associated with oxaliplatin-based chemotherapy in patients with hepatic colorectal metastases. Patients with SOS have increased postoperative morbidity after major hepatectomy, but a method for effective prevention of SOS has not been established.

Methods: Male Sprague-Dawley rats were treated with cobalt protoporphyrin IX (CoPP) or olprinone (OLP), a phosphodiesterase III inhibitor, and hepatic HO-1 expression and HO enzymatic activity were determined. Monocrotaline (MCT) was given to rats to induce SOS, and blockage of SOS by CoPP or OLP-induced hepatic HO-1 was examined in these rats. Zinc protoporphyrin IX (ZnPP), a competitive HO inhibitor, was given to MCT-treated rats together with OLP to clarify the mechanism of protection against SOS. We also examined if OLP preserved remnant liver function after 70\% hepatectomy in MCT-treated rats.

Results: OLP upregulated hepatic HO-1 protein expression and $\mathrm{HO}$ enzymatic activity, and activated Akt protein. Administration of ZnPP to OLP-treated rats resulted in inhibition of HO activity and inactivation of Akt. Induction of $\mathrm{HO}-1$ by pretreatment with $\mathrm{CoPP}$ led to amelioration of SOS in histological findings and blockage of elevation of serum liver enzymes. Pretreatment with OLP gave a similar result and preserved remnant liver function, as indicated by improved survival after hepatectomy. ZnPP completely abolished the protective effects of OLP.

Conclusions: Protection of the liver from drug-induced injury by prior upregulation of HO-1 using OLP may have potential as a therapeutic strategy for prevention of SOS. 


\section{Introduction}

Surgical resection is the standard treatment for patients with hepatic colorectal metastases (HCRM) and is only the therapy leading to cure. ${ }^{1}$ Recent regimens of neoadjuvant chemotherapy using 5-fluorouracil (5FU) and leucovorin combined with new anti-cancer drugs such as irinotecan or oxaliplatin are able to convert unresectable HCRM into resectable disease. ${ }^{2}{ }^{3}$ Therefore, chemotherapy regimens containing these new agents have become the standard of care in this setting. ${ }^{4}$ However, use of these new agents requires caution, since hepatic vascular alterations, such as sinusoidal dilatation and hemorrhage, identical with those of sinusoidal obstruction syndrome (SOS) are observed in $79 \%$ of hepatectomy specimens from patients treated with oxaliplatin-based neoadjuvant chemotherapy. ${ }^{5}$

Dietary ingestion of pyrrolizidine alkaloids and cytotoxic drugs such as azathioprine, cyclophosphamide, busulfan and dacarbazine are well known as major causes of SOS, ${ }^{6}$ and oxaliplatin also has been associated with SOS in recent years. ${ }^{78}$ The sinusoidal alterations in the liver result in bluish discoloration, edema, and a spongiform consistency similar to that of early cirrhosis. These phenomena, which are referred to as blue liver syndrome, increase potential intraoperative bleeding and decrease hepatic functional reserve, ${ }^{9}{ }^{10}$ but a method for effective prevention of SOS has not been established. Furthermore, the preoperative diagnosis of SOS after oxaliplatin-based chemotherapy is often overlooked because SOS may be present despite a normal liver function test. ${ }^{9}$ However, if hepatic resection is performed without recognition of latent liver injury, irreversible hepatic failure can develop after surgery. ${ }^{11} 12$ Additionally, once SOS occurs the sinusoidal lesions persist for several months after completion of chemotherapy regimens, ${ }^{5}$ and this may affect the timing of curative resection for HCRM. Therefore, chemotherapy-induced SOS may have a negative impact on curative resection of HCRM, despite successful tumor regression by neoadjuvant chemotherapy. This background suggests that a strategy for prevention of SOS is required to optimize the potential 
for liver resection.

The heme oxygenase (HO) enzyme system is a current area of study because of its cytoprotective properties. HO-1, the inducible isoform of the HO family, is of particular interest because it is upregulated in response to pathologic stimuli such as hypoxia, endotoxicemia, hemorrhagic shock, and drug-induced liver injury to maintain cellular function against sublethal stress in almost all organs, including the liver. ${ }^{13}$ However, no clinical trial associated with HO-1 has been reported to date. The cause of SOS is considered to be insult of sinusoidal endothelial cells (SECs) induced by chemical agents, ${ }^{8}$ and therefore protection of SECs may be the key to a preventive strategy for SOS. In the current study, we focused on olprinone (OLP), a phosphodiesterase (PDE) III inhibitor that has a protective effect on SECs in vivo. ${ }^{14}$ The purpose of the study was to investigate the pathogenesis of SOS and examine pharmacological prevention of SOS by induction of HO-1 by OLP.

\section{Materials and Methods}

\section{Reagents}

Monocrotaline (MCT) was purchased from Sigma Aldrich (St. Louis, MO). To prepare a solution of $\mathrm{MCT}$ at $10 \mathrm{mg} / \mathrm{ml}, 500 \mathrm{mg}$ of $\mathrm{MCT}$ was dissolved in $1.0 \mathrm{~N} \mathrm{HCl}$ and the $\mathrm{pH}$ was adjusted to 7.4 with $0.5 \mathrm{~N} \mathrm{NaOH} .{ }^{15} \mathrm{PBS}$ (pH 7.4) was added to increase the total volume to 50 ml. Olprinone (OLP), a phosphodiesterase (PDE) III inhibitor (Eisai Pharmaceutical Co. Tokyo, Japan), was dissolved in distilled water. Zinc protoporphyrin IX (ZnPP), competitive HO enzymatic activity inhibitor, ${ }^{16}$ and cobalt protoporphyrin IX (CoPP), HO-1 inducer, were purchased from Frontier Scientific Europe (Carnforth, UK). To prepare solutions of ZnPP at 2 $\mathrm{mg} / \mathrm{ml}$ and $\mathrm{CoPP}$ at $1 \mathrm{mg} / \mathrm{ml}$, each was dissolved in $0.2 \mathrm{~N} \mathrm{NaOH}$ and the $\mathrm{pH}$ was adjusted to 7.4 with $1 \mathrm{~N} \mathrm{HCl} .{ }^{17} \mathrm{PBS}(\mathrm{pH} 7.4$ ) was added to increase the total volume as appropriate. All other agents were purchased from Sigma. 


\section{Animal model}

Male Sprague-Dawley rats of 7-8 weeks of age and weighing 250 $\pm 50 \mathrm{~g}$ were obtained from SLC (Shizuoka, Japan). The animals had free access to food and water. The experimental protocol was approved by the Animal Research Committee of Kyoto University, and all animals received humane care according to NIH Guidelines for the Care and Use of Laboratory Animals.

Monocrotaline (MCT)-treated rats were used as an experimental model of sinusoidal obstruction syndrome (SOS); this approach has previously been reported as a reproducible model of SOS. ${ }^{18}$ Rats were fasted $12 \mathrm{~h}$ before MCT treatment, but were allowed water $\mathrm{ad}$ libitum. MCT (90 mg/kg) was administered by gavage and the rats were then allowed food and water ad libitum. A comparison between oxaliplatin-induced human SOS and MCT-induced rat SOS is shown in Figure 1. Photomicrographs of liver sections (Figs. 1A and 1C) from a 58-year-old patient with SOS were obtained by liver biopsy two weeks after completion of 6 cycles of oxaliplatin-based chemotherapy (FOLFOX6), which were performed after 3 cycles of irinotecan-based chemotherapy (FOLFIRI) for unresectable liver metastases from rectal cancer. HE staining revealed sinusoidal congestion and dilatation, coagulative necrosis of hepatocytes, and recruitment of neutrophils (Fig. 1A), and Masson-Trichrome staining showed peri-central fibrosis (Fig. 1C). Histopathological changes in rats after treatment with $90 \mathrm{mg} / \mathrm{kg}$ MCT occurred in four phases. Early manifestation of toxicity, as indicated by mild sinusoidal hemorrhage and dilation, and mild damage to the endothelium of the central vein started on day 1 . On days 2 and 3 , severe sinusoidal changes and damage to the endothelium of the central vein, coagulative necrosis of hepatocytes, and recruitment of neutrophils were observed (Fig. 1B). The histopathological changes in this phase are similar to those in human SOS. On days 4 to 6 , the histological liver insults gradually attenuated, while perivenular fibrosis was observed in areas surrounding central 
veins (Fig. 1D). After day 7, almost all animals had fully recovered. Based on these results, histopathological and biochemical analyses were performed at $48 \mathrm{~h}$ after MCT treatment, since histopathological changes in this period are most similar to those in human SOS.

\section{Assessment of hepatic HO-1 expression following pharmacological treatment}

To investigate hepatic HO-1 expression, CoPP or OLP was administered to rats. CoPP was given via a single intraperitoneal (ip) injection $(5.0 \mathrm{mg} / \mathrm{kg})$ and liver tissues were collected at $6,12,24$ and $48 \mathrm{~h}$ after CoPP treatment $(\mathrm{n}=2$ for each time point). Rats receiving

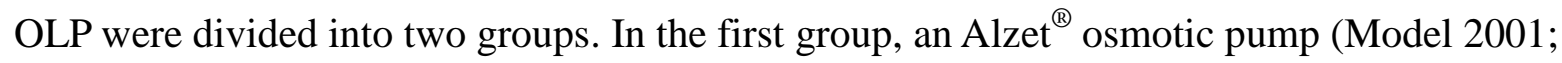
Durect Corp., Cupertino, CA, USA) was inserted into the peritoneal cavity under anesthesia with sevoflurane (Abbott Japan, Osaka, Japan) to facilitate OLP administration via continuous ip injection $(0.6 \mu \mathrm{g} / \mathrm{kg} / \mathrm{min})$. At $12 \mathrm{~h}$ after insertion of the osmotic pump, the rats also received a single ip injection of OLP $(2.0 \mathrm{mg} / \mathrm{kg})$. Rats of the second group were treated with only a single ip injection of OLP $(2.0 \mathrm{mg} / \mathrm{kg})$ without insertion of an osmotic pump. Liver tissues were obtained at $6,12,24$, and $48 \mathrm{~h}$ after the single ip injection of OLP $(\mathrm{n}=4$ for each time point) and hepatic HO-1 expression, phosphorylation of Akt, Akt, and $\mathrm{HO}$ enzymatic activity were assessed at each time point. Liver tissues were also obtained from untreated normal rats $(n=4)$ as a control.

Additionally, ZnPP was administered intraperitoneally to OLP-treated rats of the first group simultaneously with a single ip injection of OLP. Liver tissues were obtained similarly as described above.

\section{Assessment of the effect of CoPP on HO-1 in MCT-treated rats}

The animals were divided into $\underline{4}$ groups: control, MCT alone, MCT+CoPP, and $\underline{\mathrm{MCT}+\mathrm{CoPP}+\mathrm{ZnPP}}(\mathrm{n}=6$ in each group$)$. In the $\mathrm{MCT}+\mathrm{CoPP}$ group, CoPP was administered 
$12 \mathrm{~h}$ before MCT treatment via a single ip injection $(5.0 \mathrm{mg} / \mathrm{kg}) . \underline{\mathrm{In}}$ the $\mathrm{MCT}+\mathrm{CoPP}+\mathrm{ZnPP}$ group, $\mathrm{ZnPP}(50 \mu \mathrm{mol} / \mathrm{l})$ was given intraperitoneally at the same time of MCT treatment. Rats of the control group received PBS by gavage instead of MCT. At $48 \mathrm{~h}$ after MCT or PBS treatment, blood and liver samples were collected following ip injection of $40 \mathrm{mg} / \mathrm{kg}$ of pentobarbital. Serum was separated for measurement of aspartate aminotransferase (AST) and alanine aminotransferase (ALT).

\section{Assessment of the effect of OLP pretreatment on MCT-treated rats}

Rats were divided into 3 groups: MCT, MCT+OLP, and MCT+OLP+ZnPP (Fig. 2). All animals were treated with MCT as described above. In the MCT+OLP and MCT+OLP+ZnPP groups, an Alzet ${ }^{\circledR}$ osmotic pump was inserted into the peritoneal cavity $12 \mathrm{~h}$ before MCT treatment to facilitate continuous ip injection of OLP $(0.6 \mu \mathrm{g} / \mathrm{kg} / \mathrm{min})$ until the end of the protocol. A single ip injection of OLP $(2.0 \mathrm{mg} / \mathrm{kg})$ was given simultaneously with the MCT treatment. In the MCT group, distilled water was administered instead of OLP. In the $\mathrm{MCT}+\mathrm{OLP}+\mathrm{ZnPP}$ group, $\mathrm{ZnPP}(50 \mu \mathrm{mol} / \mathrm{l})$ was given via a single ip injection simultaneously with MCT treatment. Liver tissues and blood samples were collected $12 \mathrm{~h}$ after MCT treatment to investigate the expression of $\mathrm{HO}-1$ protein and $\mathrm{HO}$ enzymatic activity in each group $(\mathrm{n}=3)$, and $48 \mathrm{~h}$ after MCT treatment to assess the degree of liver injury in each group $(n=6)$

\section{Assessment of OLP pretreatment on survival of MCT-treated rats after partial}

\section{hepatectomy}

Survival in the MCT, MCT+OLP, and MCT+OLP+ZnPP groups (see previous section) was evaluated after partial hepatectomy ( $\mathrm{n}=15$ in each group). The rats underwent hepatectomy ( $70 \%$ of total liver volume) at $48 \mathrm{~h}$ after MCT treatment. The Alzet ${ }^{\circledR}$ osmotic 
pump was removed during hepatectomy to avoid inotropic effects of OLP in the postoperative period. Surgical procedures were performed under anesthesia with sevoflurane (Abbott Japan). After laparotomy through median incision, the left lateral and middle hepatic lobes were ligated and removed. During the procedure, oxygen inhalation was given via a mask and body temperature was maintained at 36.5 to $37.5^{\circ} \mathrm{C}$ using a heated table. After surgery, the rats were allowed food and water ad libitum and monitored for 10 days.

\section{Liver histology}

Liver tissues were fixed with $4 \%$ paraformaldehyde, embedded in paraffin wax and sectioned at $4 \mu \mathrm{m}$. The slides were stained with hematoxylin and eosin (HE) and histological assessment of SOS was performed blindly by a pathologist (A. M-H.). To quantify the degree of SOS, histological changes of the following three parameters were reviewed according to the scoring system described by Deleve et al. ${ }^{18}$ : endothelial damage of the central vein; coagulative necrosis of hepatocytes; and sinusoidal hemorrhage. Each parameter was graded on a 4-point scale: $0=$ absent; $1=$ mild; $2=$ moderate; $3=$ severe. To classify SOS, an overall score was determined by adding up individual scores, with a total HE score of $<2,2$ to 3,4 to 6, and 7 to 9 points classified as absent, mild, moderate and severe SOS, respectively.

\section{Immunohistochemistry}

For immunohistochemistry of $\mathrm{HO}-1$, paraffin sections were pretreated with $0.3 \% \mathrm{H}_{2} \mathrm{O}_{2}$ in methanol and then subjected to antigen retrieval in citrate buffer $(10 \mathrm{mM}, \mathrm{pH} 6.0)$ in a pressure cooker. After blocking with 3\% BSA-10\% normal serum for $1 \mathrm{~h}$, the sections were incubated with a primary antibody recognizing HO-1 (\# SPA-896; Stressgene, Victoria, Canada) at 1:200 dilution overnight at $4^{\circ} \mathrm{C}$. Subsequently, the sections were incubated with Labeled Polymer in an Envision + System HRP Kit (Dako, Tokyo, Japan) at room temperature 
for $1 \mathrm{~h}$. The sections were examined after incubation with a Liquid DAB Substrate

Chromogen System (Dako) and counterstained with hematoxylin.

In immunostaining for rat endothelial cell antigen (RECA)-1 (\#MCA-970R; Serotec,

Oxford, UK), tissue samples were directly embedded in O.C.T. compound (Sakura Finetek, Tokyo, Japan) and sectioned at $6 \mu \mathrm{m}$. The sections were fixed with $4 \%$ paraformaldehyde for $10 \mathrm{~min}$ at $4^{\circ} \mathrm{C}$. After blocking, the sections were incubated with a primary antibody recognizing RECA-1 at 1:50 dilution for $1 \mathrm{~h}$ at $4{ }^{\circ} \mathrm{C}{ }^{19}$. Immunostaining signals were visualized using Labeled Polymer in an Envision + System HRP Kit (Dako).

\section{Western blot analysis}

Tissue samples were homogenized in lysis buffer containing 50mM Tris- $\mathrm{HCl}(\mathrm{pH} 6.8)$, $10 \%$ glycerol and $2 \%$ sodium dodecylsulfate. After the concentration of the sample was determined, $0.1 \%$ bromophenol blue and 5\% 2-mercaptoethanol was added. For immunoblotting, protein $(24 \mu \mathrm{g})$ was subjected to $15 \%$ SDS-PAGE. After electrophoresis, the protein was transferred to a polyvinylidene difluoride membrane. The membranes were blocked with Blocking-One (Nacalai Tesque, Kyoto, Japan) and incubated with a primary antibody recognizing HO-1, Akt phosphorylated at $\operatorname{Ser}^{473}$ (\#9271S; Cell Signaling, Beverly, Mass), or $\alpha$-tubulin (\#CP06; Calbiochem, San Diego, CA, USA) at 1:1000 dilution overnight at $4^{\circ} \mathrm{C}$. After washing, membranes were reacted with horseradish peroxidase-conjugated antibodies (Santa Cruz Biotechnology Inc., Santa Cruz, CA, USA). Chemiluminescence was detected with Immobilon Western HRP Substrate (Millipore, Billerica, MA, USA) and the intensity of the bands was quantified with Quantity One imaging analysis software (Bio-Rad Laboratories, CA, USA). Phosphorylated Akt blot was subsequently stripped using WB stripping solution (Nakarai Tesque) at $37^{\circ} \mathrm{C}$, and was re-probed with primary antibody targeting total non-phosphorylated Akt (\#9272; Cell Signaling) to confirm equal loading of 
samples.

\section{Determination of HO enzymatic activity}

The HO enzymatic activity was measured following the method of Taylor et al. ${ }^{20}$ In brief, frozen liver tissues were homogenized on ice in $100 \mathrm{mM}$ phosphate buffer with $2 \mathrm{mM} \mathrm{MgCl}_{2}$ (HO activity buffer). After sonication, the homogenate was centrifuged at 13,000 rpm for 15 min. The supernatant $(200 \mu \mathrm{l}$, concentration of protein $25 \mathrm{mg} / \mathrm{ml})$ was dissolved in a reaction volume of $600 \mu \mathrm{l}$ of $\mathrm{HO}$ activity buffer containing $2 \mathrm{mg}$ of liver cytosol (as a source of biliverdin reductase), $0.8 \mathrm{mM}$ nicotinamide dinucleotide phosphate, $20 \mu \mathrm{M}$ hemin, $2 \mathrm{mM}$ glucose-6-phosphate, and $0.016 \mathrm{U} / \mu \mathrm{l}$ glucose-6-phosphate dehydrogenase. The reaction was performed at $37^{\circ} \mathrm{C}$ for $1 \mathrm{~h}$ in the dark and stopped by addition of $600 \mu \mathrm{l}$ of chloroform. After extraction of bilirubin, the chloroform layer was measured at $464 \mathrm{~nm}$ minus the background at $530 \mathrm{~nm}$. Based on the protein content in the reaction volume, the results are expressed as formation of bilirubin (pmol) per milligram of protein within $1 \mathrm{~h}$. Spleen tissue of control rats served as a positive control.

\section{Statistical analysis}

Statistical analysis was performed using SPSS v. 11.0.1 (SPSS Inc. IL, USA). Data are expressed as means \pm SD. Differences in measured variables between each group were assessed using a Mann-Whitney $U$ test. Survival curves from the time of partial hepatectomy were calculated using the Kaplan-Meier method and analyzed by log-rank test. $P<0.05$ was considered to indicate statistical significance.

\section{Results}




\section{activity}

The time course of HO-1 protein expression in the liver after CoPP and OLP treatment was first determined in normal rats. As shown in Fig. $\underline{3}$ A, hepatic HO-1 protein was barely detectable in the liver in untreated normal rats (control), but was detected $6 \mathrm{~h}$ after CoPP treatment and continued to increase until $24 \mathrm{~h}$. A combination of single and continuous ip injection of OLP also led to an increase in hepatic HO-1 protein expression that reached approximately 2.5 -fold higher than that in control animals at $6 \mathrm{~h}$ after the single ip injection of OLP, and was subsequently sustained at this level until $24 \mathrm{~h}$ (Fig. $\underline{3}$ B). Although HO-1 expression was decreased compared with those of previous time points at $48 \mathrm{~h}$ after the single ip injection of OLP, it was significantly higher than that in control animals. In contrast, a single ip injection of OLP alone failed to increase HO-1 protein expression at any time points. Phosphorylated Akt protein was observed at $6 \mathrm{~h}$ after a single ip injection of OLP, and kept increased throughout a continuous injection of OLP (Fig. 3B). OLP treatment led to a strong increase of hepatic HO enzymatic activity that was approximately 3-fold higher than that in control animals at all time points, while ZnPP, a competitive inhibitor of heme oxygenase, ${ }^{16}$ cancelled OLP-induced increase in HO activity in the liver (Fig. 3D) without affecting expression level of HO-1 protein (Fig. 3B, top vs. 3C, top). ZnPP also cancelled phosphorylation of Akt induced by OLP (Fig. 3B, 2nd panel vs. 3C, 2nd panel), suggesting that phosphorylation of Akt is dependent on $\mathrm{HO}$ enzymatic activity. Immunohistochemical analysis indicated that HO-1 was localized in non-parenchymal cells of liver obtained from control rats (Fig. $\underline{3 \mathrm{E})}$. In contrast, HO-1 was markedly increased in hepatocytes mainly located in the region around the central vein after CoPP or OLP treatment (Figs. $\underline{3 F}$ and G).

\section{Attenuation of SOS by cobalt protoporphyrin IX via induction of heme oxygenase-1}

In rats treated with MCT alone, macroscopic findings showed accumulation of bloody 
ascites and the color of the liver surface had turned dark-red, indicating liver congestion, at 48 $\mathrm{h}$ after MCT treatment. Histologically, sinusoidal hemorrhage and dilatation, endothelial damage of central veins, and coagulative necrosis of hepatocytes were observed predominantly in the mid- and centrilobular region (Fig. $\underline{4 \mathrm{C}}$ ). The mean score for HE staining indicated the presence of severe SOS, and excessive elevation of serum liver enzymes was observed (Table 1). RECA-1 protein expression in the liver was markedly reduced after MCT

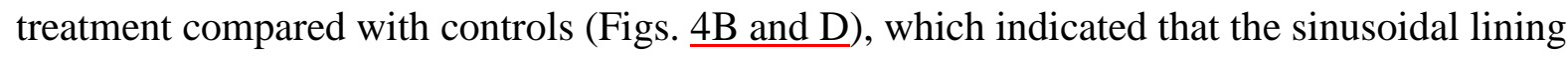
had largely disappeared. In contrast, in animals treated with MCT+CoPP, bloody ascites had disappeared and the color of the liver surface was brown, similarly to controls. HE staining indicated little sinusoidal dilation and hemorrhage, endothelial damage of central veins, or coagulative necrosis of hepatocytes (Fig. $\underline{4 \mathrm{E}}$ ), and the mean score for HE staining was significantly lower than that after MCT treatment alone and indicated mild SOS. Immunohistochemical analysis showed that the level of RECA-1 protein expression in the liver of animals in the MCT+CoPP group was comparable to that in control rats (Fig. $\underline{4 F}$ ), and pretreatment with CoPP led to a profound decrease in serum liver enzymes (AST and ALT) compared with MCT alone $(P<0.01)$. ZnPP abolished the protective effects of CoPP, as demonstrated by sinusoidal hemorrhage and dilation, endothelial damage of central veins, and coagulative necrosis of hepatocytes in the liver (Fig. 4G). The mean score for SOS was $\underline{\text { significantly higher in } \mathrm{MCT}+\mathrm{CoPP}+\mathrm{ZnPP} \text { group than that in } \mathrm{MCT}+\mathrm{CoPP} \text { group }(P<0.01)}$ and nearly as high as that in MCT group (Table 1). Immunohistochemical analysis indicated a marked decrease in RECA-1 expression in SECs (Fig. 4H), and serum biochemistry showed excessive elevation of liver enzymes (Table 1) in $\mathrm{MCT}+\mathrm{CoPP}+\mathrm{ZnPP}$ group.

\section{Olprinone inhibits MCT-induced SOS in an HO-1-dependent manner}

The effect of olprinone on SOS was examined using the protocol shown in Fig. 1. In the 
MCT group, expression of HO-1 protein was low at $12 \mathrm{~h}$ after MCT treatment, whereas overexpression of $\mathrm{HO}-1$ was observed in the MCT+OLP and MCT+OLP+ZnPP groups (Fig. 5A). However, phosphorylation of Akt and upregulation of HO enzymatic activity compared with controls was observed only in the MCT+OLP group at $12 \mathrm{~h}$ after MCT treatment (Figs. $\underline{5 \mathrm{~A} \text { and } \mathrm{B}})$. No evidence of SOS or elevation of liver enzymes was observed at $12 \mathrm{~h}$ in any group (data not shown).

In the MCT group, macro- and microscopic findings at $48 \mathrm{~h}$ after MCT treatment indicated liver congestion and severe SOS (Figs $\underline{6 \mathrm{~A} \text { and B}}$ ), which were quite similar with those of rats treated with MCT alone (Figs $\underline{4 \mathrm{C} \text { and D)}}$. In contrast, pretreatment with OLP led to elimination of ascites formation and liver congestion, and HE staining indicated little sinusoidal alteration, endothelial damage of central veins, or coagulative necrosis of hepatocytes (Fig. 6C). The mean score for HE staining was significantly lower than that in the MCT group $(P<0.01)$ and indicated that SOS was absent. Immunohistochemistry indicated abundant expression of RECA-1 protein, suggesting that the sinusoidal lining was intact (Fig. 6D). Elevation of liver enzymes was also blocked significantly by pretreatment with OLP (Table 2). These results were quite similar to those for MCT+CoPP treatment.

In MCT+OLP+ZnPP group, macroscopically, bloody ascites and liver congestion were observed, and HE staining revealed sinusoidal hemorrhage and dilation, endothelial damage of central veins, and coagulative necrosis of hepatocytes in the liver (Fig. $\underline{6 \mathrm{E}})$. The mean score for HE staining indicated severe SOS, and was significantly higher than that for the MCT+OLP group $(P<0.01)$ and nearly as high as that for the MCT group (Table 2). Immunohistochemical analysis indicated a marked decrease in RECA-1 expression in SECs (Fig. 6F), and serum biochemistry showed excessive elevation of liver enzymes (Table 2). Taken together, ZnPP treatment completely abolished the protective effects of OLP. 


\section{Preservation of remnant liver function by olprinone}

Kaplan-Meier curves for 10-day survival after partial hepatectomy are shown in Fig. . The survival rate after hepatectomy in the MCT+OLP group was significantly higher than that in the MCT group $(86.7 \%$ vs. $6.7 \%, \mathrm{P}<0.01)$. Blockage of $\mathrm{HO}$ activity by $\mathrm{ZnPP}$ resulted in a poor survival rate $(13.3 \%)$ that did not differ significantly from the rate for the MCT group and was significantly lower than the rate for the MCT+OLP group $(\mathrm{P}<0.01)$, indicating that $\mathrm{ZnPP}$ completely eliminated the preservation of remnant liver function by OLP.

\section{Discussion}

Since the first report of SOS in Jamaican patients who had ingested pyrrolizidine alkaloids, it has emerged that various pharmacological agents, including oxaliplatin, cause SOS. In the current study, we used a rat model of monocrotaline (MCT)-induced SOS to search for a therapeutic strategy for prevention of SOS. Monocrotaline is a pyrrolizidine alkaloid found in Crotalari, and the MCT-induced SOS rat model has the same histological characteristics as the human disease, including sinusoidal dilation and hemorrhage, endothelial damage of central veins, and necrotic hepatocytes in the liver, as well as similar "clinical features" of hyperbilirubinemia, hepatomegaly, and ascites formation. ${ }^{21}$

The mechanism of MCT-induced SOS has been examined in vitro and in vivo, as described by Deleve et al. ${ }^{8}$ The first step of SOS is SEC-specific injury induced by the reactive metabolite of MCT. Subsequently, morphological alteration of SECs and degradation of the sinusoidal lining due to matrix metalloproteinases (MMPs) released from SECs occur $12 \mathrm{~h}$ after MCT treatment and result in disturbance of the hepatic microcirculation. ${ }^{8}$ Prospective studies for prevention of SOS have been conducted from the perspective of improvement of hepatic microcirculation using prostaglandin E1 or heparin, but these drugs do not reduce the incidence of fatal SOS. ${ }^{22}$ Hence, treatment of dysfunction of the hepatic 
microcirculation due to SEC damage is insufficient to overcome SOS.

In contrast, pretreatment with MMP inhibitors started $24 \mathrm{~h}$ before MCT treatment led to amelioration of MCT-induced rat SOS. ${ }^{23}$ Furthermore, in a recent retrospective study, Ribero et al. demonstrated that addition of bevacizumab, a recombinant human monoclonal antibody to vascular endothelial growth factor (VEGF)-A, in 5FU-plus-oxaliplatin chemotherapy led to reduction of the incidence and severity of oxaliplatin-induced SOS. ${ }^{24}$ The mechanism of a bevacizumab-mediated hepatoprotection was suggested to be due to VEGF blockage, which may attenuate damage to SECs by downregulating MMP production. Therefore, protection against SEC damage may offer a preventive strategy for SOS. In this study, however, CoPP or OLP do not attenuate SOS with simultaneous administration with MCT (data not shown). In contrast, prophylactic upregulation of HO-1 by pretreatment with CoPP or OLP was effective for blockage of SOS, and collectively the current and previous data indicate that prophylactic protection of SECs has therapeutic potential for prevention of SOS.

HO-1 is the rate-limiting enzyme of heme catabolism, catalyzing the breakdown of heme into biliverdin, iron, and carbon monoxide. ${ }^{25}$ Recent studies have demonstrated that HO-1 expression and associated metabolites have cytoprotective properties including anti-apoptotic effects through activation of the Akt signaling pathway, ${ }^{26}$ anti-inflammatory effects due to inhibition of production of inflammatory cytokines, ${ }^{27}$ and anti-oxidant effects via an increase in expression and catalytic activity of superoxide dismutase in vascular endothelial cells. ${ }^{28} \mathrm{In}$ the current study, prophylactic upregulation of HO-1 by CoPP maintained the integrity of SECs in MCT-treated rats, as indicated by sustained RECA-1 protein expression, and this resulted in inhibition of the occurrence of SOS. These results suggest that HO-1 may have therapeutic potential for various diseases, but to date no clinical trials associated with HO-1 have been reported. Most in vivo studies have shown beneficial effects of CoPP-induced HO-1 expression, ${ }^{29,28}$ but side effects observed in animal studies have made it difficult to use 
CoPP in a clinical setting. ${ }^{30}$ Therefore, we examined induction of HO-1 expression using olprinone (OLP), a drug that has been proven to be safe for clinical applications.

OLP is a new selective PDE III inhibitor with combined positive inotropic and vasodilating properties mediated through elevation of intracellular cyclic adenosine monophosphate (cAMP) levels in vascular smooth muscle cells and cardiomyocytes by preventing degradation of $\mathrm{CAMP} .{ }^{31}$ Abundant PDE III is present in the liver, ${ }^{32}$ and in vivo studies using PDE III inhibitors have shown beneficial effects on hepatic ischemia-reperfusion injury. ${ }^{14,33}$ However, the precise mechanism by which PDE III inhibitors protect the liver remains unclear. In the current study, we showed a correlation of cytoprotective properties of a PDE III inhibitor and HO-1, since treatment with OLP (similarly to CoPP) led to overexpression of $\mathrm{HO}-1$ protein and upregulation of $\mathrm{HO}$ enzymatic activity in the liver in the presence or absence of MCT. Furthermore, we found that OLP activated (phosphorylated) Akt, which is known as a major regulator of cell survival and reported to protect mitochondrial membrane potential from reactive oxygen species and inactivates proapoptotic protein. ${ }^{34}$ ZnPP abolished the activation of Akt, indicating that OLP-induced activation of Akt was mediated by HO. Pretreatment with OLP protects SECs from MCT toxicity, as shown by maintenance of RECA-1 protein expression, and inhibits the occurrence of SOS, as indicated by the absence of histopathological features of SOS and the lack of elevation of plasma liver enzymes. Furthermore, blockage of HO activity by administration of ZnPP abolished OLP-mediated phosphorylation of Akt and canceled hepatoprotection in OLP-pretreated rats. These results suggest that OLP-induced HO-1 exerts its cytoprotective property through activation of survival signaling pathway, Akt, and the hepatoprotective effect of OLP is approximately equal to that of CoPP, despite reduced upregulation of expression of HO-1 by OLP compared to CoPP.

Vauthey et al reported that oxaliplatin was associated with an increase in sinusoidal injury 
but no increase in perioperative morbidity or mortality, ${ }^{7}$ whereas Nakano et al reported sinusoidal injury induced by oxaliplatin-contained chemotherapy resulted in a poor liver functional reserve as shown by a high value of preoperative ICG-R15 and was associated with higher morbidity and longer hospital stay in patients presenting with sinusoidal injury. ${ }^{10}$ Therefore, strategy for preserving liver functional reserve should be required to perform safe liver resection in patients received oxaliplatin-contained chemotherapy. In the present study, we showed that pretreatment with OLP gave a significant improvement in survival rate after hepatic resection in MCT-treated rats. This result suggests that OLP treatment not only blocks SOS histopathologically, but also preserves remnant liver function after major hepatectomy. OLP has been widely used in various clinical situations and may be promising for inhibition of SOS induced by chemotherapy. However, a recent study indicated that HO-1 has a potential progressive role in cancer cells,,$\frac{35}{3}$ and the effect of PDE III inhibitors on cancer cells remains unknown. Therefore, further investigation will be necessary before clinical application of OLP in patients with HCRM.

In summary, the current results show that prophylactic upregulation of HO-1 by CoPP or olprinone is effective for maintenance of the sinusoidal lining in SECs and blockage of MCT-induced SOS. Pretreatment with OLP also led to a significant improvement in survival rate after hepatectomy in MCT-treated rats. These results suggest that OLP may offer a therapeutic strategy for preventing SOS by protection of SECs from drug-induced injury through prior upregulation of a cytoprotective gene. 


\section{References}

1. Lochan R, White SA, Manas DM. Liver resection for colorectal liver metastasis. Surg Oncol 2007; 16(1):33-45.

2. Adam R, Delvart V, Pascal G, et al. Rescue surgery for unresectable colorectal liver metastases downstaged by chemotherapy: a model to predict long-term survival. Ann Surg 2004; 240(4):644-57; discussion 657-8.

3. Giacchetti S, Itzhaki M, Gruia G, et al. Long-term survival of patients with unresectable colorectal cancer liver metastases following infusional chemotherapy with 5-fluorouracil, leucovorin, oxaliplatin and surgery. Ann Oncol 1999; 10(6):663-9.

4. Zorzi D, Laurent A, Pawlik TM, et al. Chemotherapy-associated hepatotoxicity and surgery for colorectal liver metastases. Br J Surg 2007; 94(3):274-86.

5. Rubbia-Brandt L, Audard V, Sartoretti P, et al. Severe hepatic sinusoidal obstruction associated with oxaliplatin-based chemotherapy in patients with metastatic colorectal cancer. Ann Oncol 2004; 15(3):460-6.

6. Coppell JA, Brown SA, Perry DJ. Veno-occlusive disease: cytokines, genetics, and haemostasis. Blood Rev 2003; 17(2):63-70.

7. Vauthey JN, Pawlik TM, Ribero D, et al. Chemotherapy regimen predicts steatohepatitis and an increase in 90-day mortality after surgery for hepatic colorectal metastases. J Clin Oncol 2006; 24(13):2065-72.

8. DeLeve LD. Hepatic microvasculature in liver injury. Semin Liver Dis 2007; 27(4):390-400.

9. Bilchik AJ, Poston G, Curley SA, et al. Neoadjuvant chemotherapy for metastatic colon cancer: a cautionary note. J Clin Oncol 2005; 23(36):9073-8.

10. Nakano H, Oussoultzoglou E, Rosso E, et al. Sinusoidal injury increases morbidity after major hepatectomy in patients with colorectal liver metastases receiving 
preoperative chemotherapy. Ann Surg 2008; 247(1):118-24.

11. Ishizaki T, Abe T, Koyanagi Y, et al. [A case of liver failure associated with liver damage due to mFOLFOX 6 after resection for multiple liver metastases from colorectal cancer]. Gan To Kagaku Ryoho 2007; 34(6):945-8.

12. Schouten van der Velden AP, Punt CJ, Van Krieken JH, et al. Hepatic veno-occlusive disease after neoadjuvant treatment of colorectal liver metastases with oxaliplatin: A lesson of the month. Eur J Surg Oncol 2007.

13. Farombi EO, Surh YJ. Heme oxygenase-1 as a potential therapeutic target for hepatoprotection. J Biochem Mol Biol 2006; 39(5):479-91.

14. Kume M, Banafsche R, Yamamoto Y, et al. Dynamic changes of post-ischemic hepatic microcirculation improved by a pre-treatment of phosphodiesterase-3 inhibitor, milrinone. J Surg Res 2006; 136(2):209-18.

15. Prie S, Stewart DJ, Dupuis J. EndothelinA receptor blockade improves nitric oxide-mediated vasodilation in monocrotaline-induced pulmonary hypertension. Circulation 1998; 97(21):2169-74.

16. Labbe RF, Vreman HJ, Stevenson DK. Zinc protoporphyrin: A metabolite with a mission. Clin Chem 1999; 45(12):2060-72.

17. Amersi F, Buelow R, Kato H, et al. Upregulation of heme oxygenase-1 protects genetically fat Zucker rat livers from ischemia/reperfusion injury. J Clin Invest 1999; 104(11):1631-9.

18. DeLeve LD, McCuskey RS, Wang X, et al. Characterization of a reproducible rat model of hepatic veno-occlusive disease. Hepatology 1999; 29(6):1779-91.

19. Graupera M, March S, Engel P, et al. Sinusoidal endothelial COX-1-derived prostanoids modulate the hepatic vascular tone of cirrhotic rat livers. Am J Physiol Gastrointest Liver Physiol 2005; 288(4):G763-70. 
20. Taylor JL, Carraway MS, Piantadosi CA. Lung-specific induction of heme oxygenase-1 and hyperoxic lung injury. Am J Physiol 1998; 274(4 Pt 1):L582-90.

21. DeLeve LD, Shulman HM, McDonald GB. Toxic injury to hepatic sinusoids: sinusoidal obstruction syndrome (veno-occlusive disease). Semin Liver Dis 2002; 22(1):27-42.

22. Helmy A. Review article: updates in the pathogenesis and therapy of hepatic sinusoidal obstruction syndrome. Aliment Pharmacol Ther 2006; 23(1):11-25.

23. Deleve LD, Wang X, Tsai J, et al. Sinusoidal obstruction syndrome (veno-occlusive disease) in the rat is prevented by matrix metalloproteinase inhibition.

Gastroenterology 2003; 125(3):882-90.

24. Ribero D, Wang H, Donadon M, et al. Bevacizumab improves pathologic response and protects against hepatic injury in patients treated with oxaliplatin-based chemotherapy for colorectal liver metastases. Cancer 2007; 110(12):2761-7.

25. Maines MD. The heme oxygenase system: a regulator of second messenger gases. Annu Rev Pharmacol Toxicol 1997; 37:517-54.

26. Olszanecki R, Rezzani R, Omura S, et al. Genetic suppression of HO-1 exacerbates renal damage: reversed by an increase in the antiapoptotic signaling pathway. Am J Physiol Renal Physiol 2007; 292(1):F148-57.

27. Song R, Kubo M, Morse D, et al. Carbon monoxide induces cytoprotection in rat orthotopic lung transplantation via anti-inflammatory and anti-apoptotic effects. Am J Pathol 2003; 163(1):231-42.

28. Turkseven S, Kruger A, Mingone CJ, et al. Antioxidant mechanism of heme oxygenase-1 involves an increase in superoxide dismutase and catalase in experimental diabetes. Am J Physiol Heart Circ Physiol 2005; 289(2):H701-7.

29. L'Abbate A, Neglia D, Vecoli C, et al. Beneficial effect of heme oxygenase-1 
expression on myocardial ischemia-reperfusion involves an increase in adiponectin in mildly diabetic rats. Am J Physiol Heart Circ Physiol 2007; 293(6):H3532-41.

30. Schmidt R. Cobalt protoporphyrin as a potential therapeutic agent? Faseb J 2007; 21(11):2639; author reply 2640.

31. Mizushige K, Ueda T, Yukiiri K, Suzuki H. Olprinone: a phosphodiesterase III inhibitor with positive inotropic and vasodilator effects. Cardiovasc Drug Rev 2002; 20(3):163-74.

32. Manganiello VC, Degerman E, Taira M, et al. Type III cyclic nucleotide phosphodiesterases and insulin action. Curr Top Cell Regul 1996; 34:63-100.

33. Kobayashi T, Sugawara Y, Ohkubo T, et al. Effects of amrinone on hepatic ischemia-reperfusion injury in rats. J Hepatol 2002; 37(1):31-8.

34. Tapodi A, Debreceni B, Hanto K, et al. Pivotal role of Akt activation in mitochondrial protection and cell survival by poly(ADP-ribose)polymerase-1 inhibition in oxidative stress. J Biol Chem 2005; 280(42):35767-75.

35. Berberat PO, Dambrauskas Z, Gulbinas A, et al. Inhibition of heme oxygenase-1 increases responsiveness of pancreatic cancer cells to anticancer treatment. Clin Cancer Res 2005; 11(10):3790-8. 


\section{Figure Legends}

Figure 1: Liver sections of human SOS (left column, A and C) and sections taken from rats after treatment with $90 \mathrm{mg} / \mathrm{kg} \mathrm{MCT} \mathrm{(right} \mathrm{column,} \mathrm{B} \mathrm{and} \mathrm{D).} \mathrm{HE} \mathrm{staining} \mathrm{(A} \mathrm{and} \mathrm{B,} \mathrm{400x)}$ revealed sinusoidal congestion and dilatation. The arrowheads indicate the area of coagulative $\underline{\text { necrosis of hepatocytes. Masson-Trichrome staining (C and D, 400x) showed peri-central }}$ fibrosis.

Figure 2: Experimental protocol. In all animals, an osmotic pump was inserted into the peritoneal cavity under anesthesia with sevoflurane to permit continuous ip injection of OLP or distilled water. At $12 \mathrm{~h}$ after insertion of the osmotic pump, MCT (90 mg/kg) was administered by gavage and a single ip injection of distilled water, OLP, or OLP and ZnPP was given. Blood and liver samples were collected at 12 and 48 hours after MCT treatment. MCT: monocrotaline, ip: intraperitoneal, OLP: olprinone, ZnPP: zinc protoporphyrin IX. $\underline{\text { Figure 3: }}$ : Western blotting analysis of hepatic HO-1 and $\alpha$-tubulin, and HO-1, phosphorylated

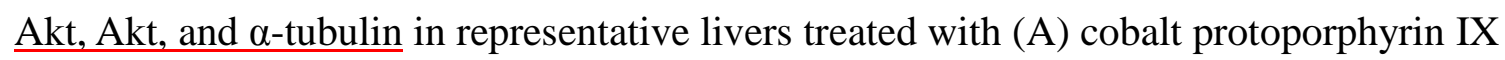
(CoPP) and (B) olprinone (OLP), respectively. Relative HO-1 protein levels were quantified by densitometry. Data are expressed as means $\pm \mathrm{SD}(\mathrm{n}=4, * \mathrm{P}<0.01$ vs. control; $* * \mathrm{P}<0.05$ vs. control). Control indicates an untreated normal rat. (C) Western blotting analysis of hepatic HO-1 and $\alpha$-tubulin, and HO-1, phosphorylated Akt, Akt, and $\alpha$-tubulin in $\underline{\text { representative livers treated with OLP }+\mathrm{ZnPP}} \underline{(\mathrm{D})} \mathrm{HO}$ enzymatic activity in rat livers from $\underline{\text { OLP-treated rats and OLP }+Z n P P-t r e a t e d ~ r a t s . ~ D a t a ~ a r e ~ e x p r e s s e d ~ a s ~ m e a n s ~} \pm \mathrm{SD}(\mathrm{n}=4, \uparrow \mathrm{P}<$ 0.01 vs. control; $\uparrow \uparrow \mathrm{P}<0.05$ vs. control; $\$ \mathrm{P}<0.05$ vs. OLP treatment at each time). Liver sections from control $\underline{(\mathrm{E})}$, OLP-treated $\underline{(\mathrm{F})}$, and CoPP-treated $\underline{(\mathrm{G})}$ rats were subjected to immunohistochemical analysis to determine expression patterns of HO-1 protein. HO-1: heme oxygenase-1, OLP: olprinone, CoPP: cobalt protoporphyrin IX.

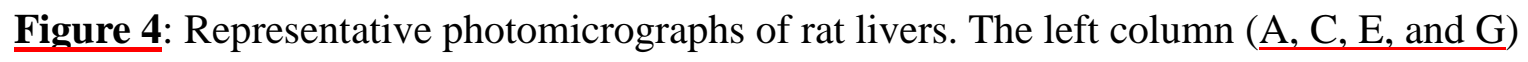


shows HE staining (x 400) and the right column ( $\underline{B, D, F}$, and $\mathrm{H})$ shows

immunohistochemistry of RECA-1 (x 400). (A) In the control group there was no evidence of sinusoidal alteration, endothelial damage of central veins, or coagulative necrosis of hepatocytes. (B) Immunohistochemical analysis in the control group indicated RECA-1 protein expression in the sinusoidal lining. (C) Treatment with MCT alone resulted in severe sinusoidal hemorrhage, endothelial damage of central veins, and coagulative necrosis of hepatocytes. (D) With MCT alone, RECA-1 protein expression was significantly reduced compared with that in the control group. (E) Induction of HO-1 by pretreatment with CoPP led to amelioration of pathohistological changes in the liver induced by MCT treatment. (F) Induction of HO-1 also restored RECA-1 protein expression to the level in the control group. ( $\mathrm{G}$ and $\mathrm{H})$ However, inhibition of $\mathrm{HO}$ activity by $\mathrm{ZnPP}$ canceled CoPP-provided hepatoprotective effect. MCT: monocrotaline, CoPP: cobalt protoporphyrin IX, RECA-1: rat endothelial cell antigen-1.

Figure 5: (A) Western blotting analysis of hepatic HO-1, phosphorylated Akt, and Akt protein in representative livers obtained from control (untreated) and treated groups at $12 \mathrm{~h}$ after treatment. Relative HO-1 protein levels were quantified by densitometry. Data are expressed as means $\pm \mathrm{SD}(\mathrm{n}=3, * \mathrm{P}<0.01$ vs. control). (B) $\mathrm{HO}$ enzymatic activity in rat livers from each group at $12 \mathrm{~h}$ after treatment. Data are expressed as means $\pm \mathrm{SD}(\mathrm{n}=3, \dagger \mathrm{P}<0.01$ vs. $\mathrm{MCT}+\mathrm{OLP}+\mathrm{ZnPP})$.

Figure 6: Representative photomicrographs of rat livers. The left column (A, C and E) shows HE staining (x 400) and the right column ( $\mathrm{B}, \mathrm{D}$ and $\mathrm{F}$ ) shows immunohistochemistry of RECA-1 (x 400). (A and B) In MCT group, severe sinusoidal hemorrhage, endothelial damage of central veins, and coagulative necrosis of hepatocytes were observed, together with significant reduction of RECA-1 protein expression. (C and D) Pretreatment with OLP led to blockage of sinusoidal changes induced by MCT treatment and increased RECA-1 protein 
expression compared with $\mathrm{MCT}$ group. (E and $\mathrm{F}$ ) $\mathrm{ZnPP}$ treatment completely inhibited the protective effects of pretreatment with OLP.

Figure 7: Kaplan-Meier curves for 10-day survival after partial hepatectomy. Full line ( - ), broken line ( - - ), and dotted line ( $\cdots \cdots \cdot \cdots)$, indicate survival curves of MCT, MCT+OLP and $\mathrm{MCT}+\mathrm{OLP}+\mathrm{ZnPP}$, respectively (10-day survival rates: MCT, 6.7\%; MCT+OLP, 86.7\%; $\mathrm{MCT}+\mathrm{OLP}+\mathrm{ZnPP}, 13.3 \%$ ). The survival rate in MCT+OLP was significantly higher than in MCT and MCT+OLP+ZnPP (P < 0.01). RECA-1: rat endothelial cell antigen-1, HO: heme oxygenase. 
Table 1. SOS score for HE staining and serum biochemistry after CoPP administration

\begin{tabular}{ccccc} 
Item & Control & MCT alone & MCT + CoPP & $\underline{\text { MCT }+ \text { CoPP }+\mathrm{ZnPP}}$ \\
\hline SOS score & 0 & $7.5 \pm 1.4$ & $2.8 \pm 0.5 *$ & $\underline{8.3 \pm 1.2 \dagger}$ \\
AST (IU/l) & $87.4 \pm 33.2$ & $3300.0 \pm 991.0$ & $196.6 \pm 83.2 *$ & $\underline{3498.8 \pm 644.2 \dagger}$ \\
ALT (IU/1) & $48.4 \pm 10.2$ & $2072.0 \pm 882.5$ & $163.4 \pm 73.1 *$ & $\underline{1846.8 \pm 245.4 \dagger}$ \\
\hline
\end{tabular}

Data are shown as means \pm SD. $* \mathrm{P}<0.01$ compared with MCT alone. $₫ \mathrm{P}<0.01$ compared with MCT + CoPP. Control indicates rats treated with PBS instead of MCT.

MCT, monocrotaline; CoPP, cobalt protoporphyrin IX; SOS, sinusoidal obstruction syndrome; AST, aspartate aminotransferase; ALT, alanine aminotransferase 
Table 2. SOS score for HE staining and serum biochemistry after OLP administration

\begin{tabular}{cccc} 
Item & MCT & MCT + OLP & MCT+OLP+ZnPP \\
\hline SOS score & $7.7 \pm 1.5$ & $1.8 \pm 1.0 *$ & $7.8 \pm 1.9 \dagger$ \\
AST (IU/l) & $3752.5 \pm 1172.8$ & $269.7 \pm 162.0 *$ & $5658.3 \pm 3762.6 \dagger$ \\
ALT (IU/l) & $2466.3 \pm 933.1$ & $146.8 \pm 69.8 *$ & $2778.3 \pm 1636.2 \dagger$ \\
\hline
\end{tabular}

Data are shown as means $\pm \mathrm{SD} . * \mathrm{P}<0.01$ compared with $\mathrm{MCT}, \dagger \mathrm{P}<0.01$ compared with MCT+OLP. MCT, monocrotaline; OLP, olprinone; ZnPP, zinc protoporphyrin IX; SOS, sinusoidal obstruction syndrome; AST, aspartate aminotransferase; ALT, alanine aminotransferase 


\section{Figure 1}

\section{Human SOS}

A

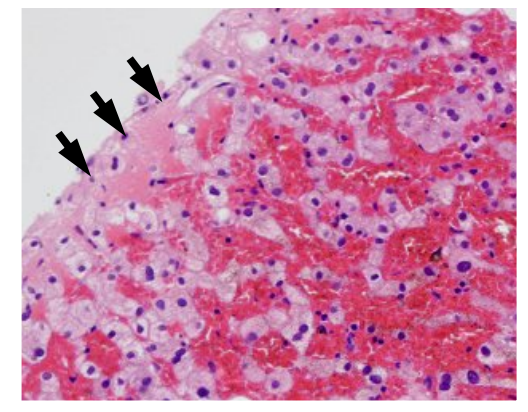

C

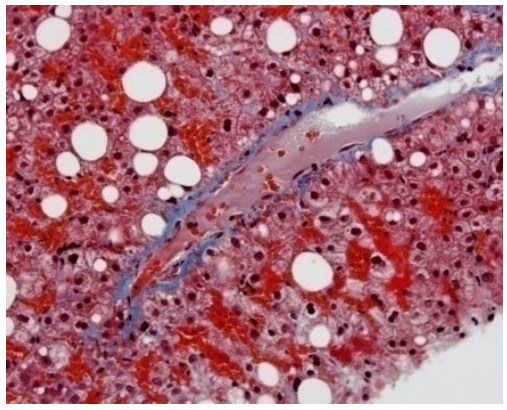

MCT-induced SOS

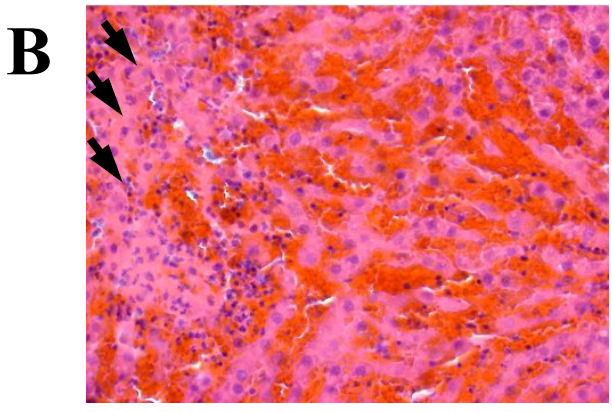

D

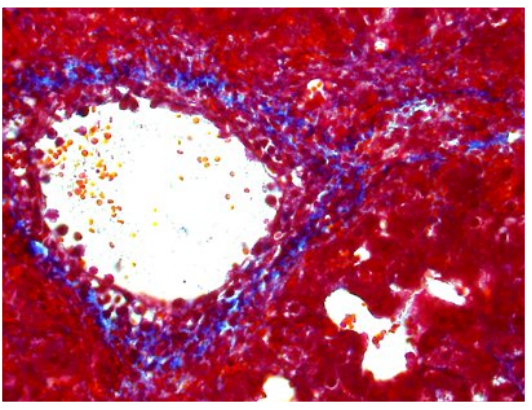




\section{Figure 2}

MCT
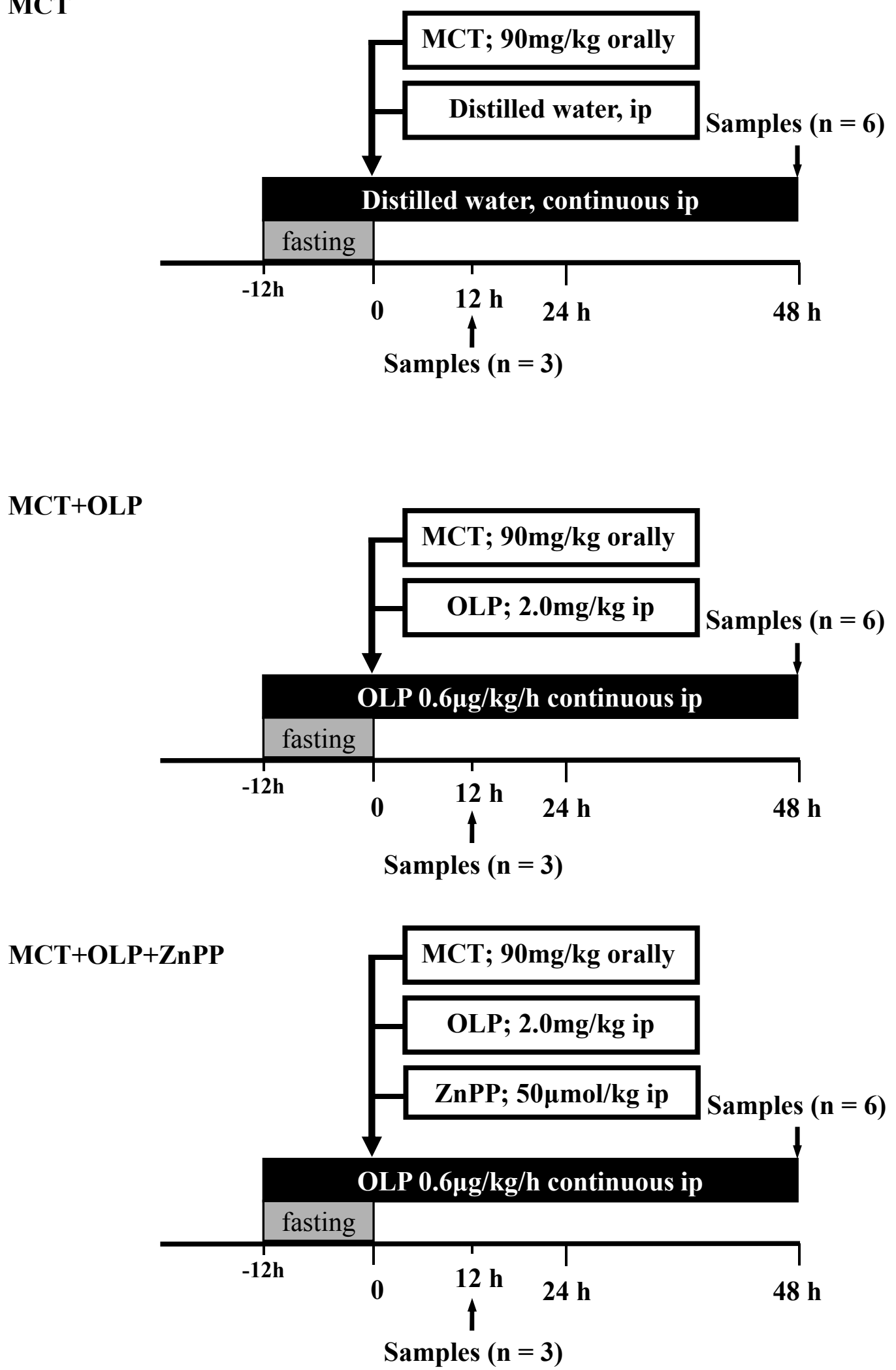


\section{Figure 3}

A

$$
\begin{array}{r}
\text { Time after } \\
\text { CoPP treatment } \\
\text { HO-1 }
\end{array}
$$

$$
\text { o-tubulin }
$$

$$
\text { Control } \quad 6 \mathrm{~h} \quad 12 \mathrm{~h} \quad 24 \mathrm{~h} \quad 48 \mathrm{~h}
$$

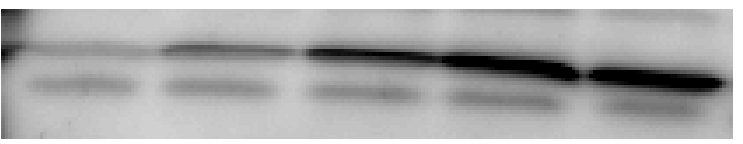

B

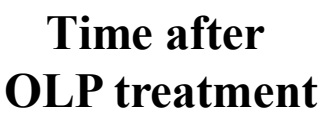

$$
\text { Control } \quad 6 \mathrm{~h} \quad 12 \mathrm{~h} \quad 24 \mathrm{~h} \quad 48 \mathrm{~h}
$$

HO-1

Phosphorylated Akt

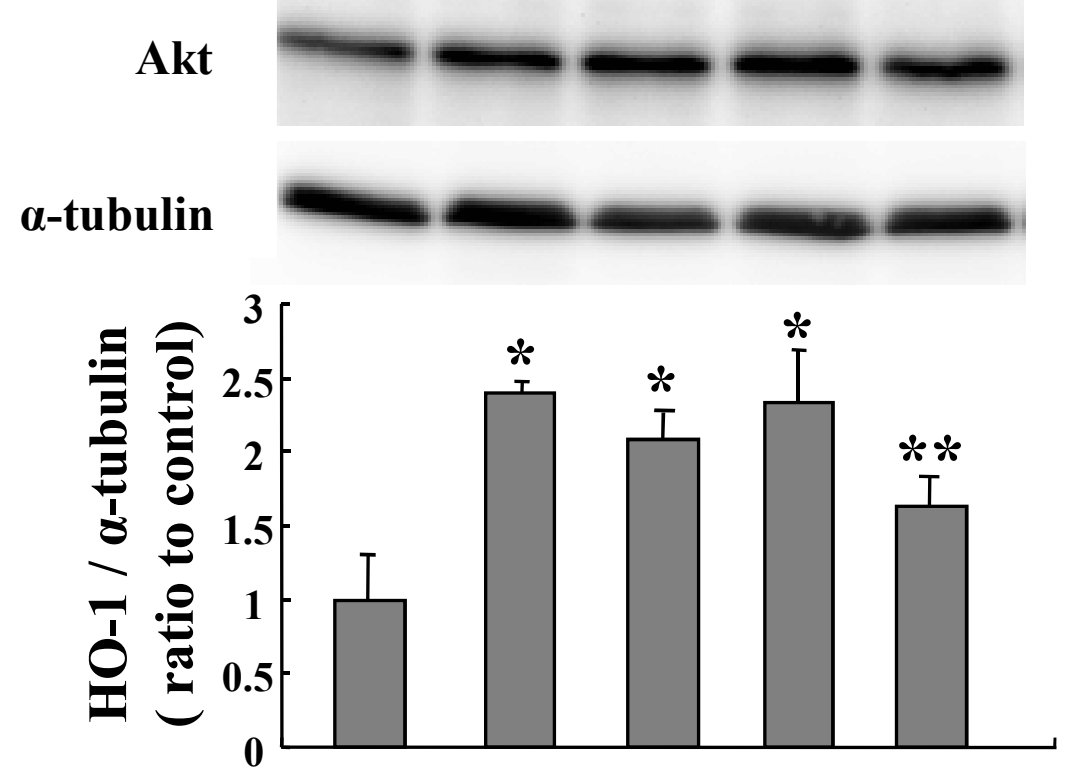




\section{Figure 3}

C

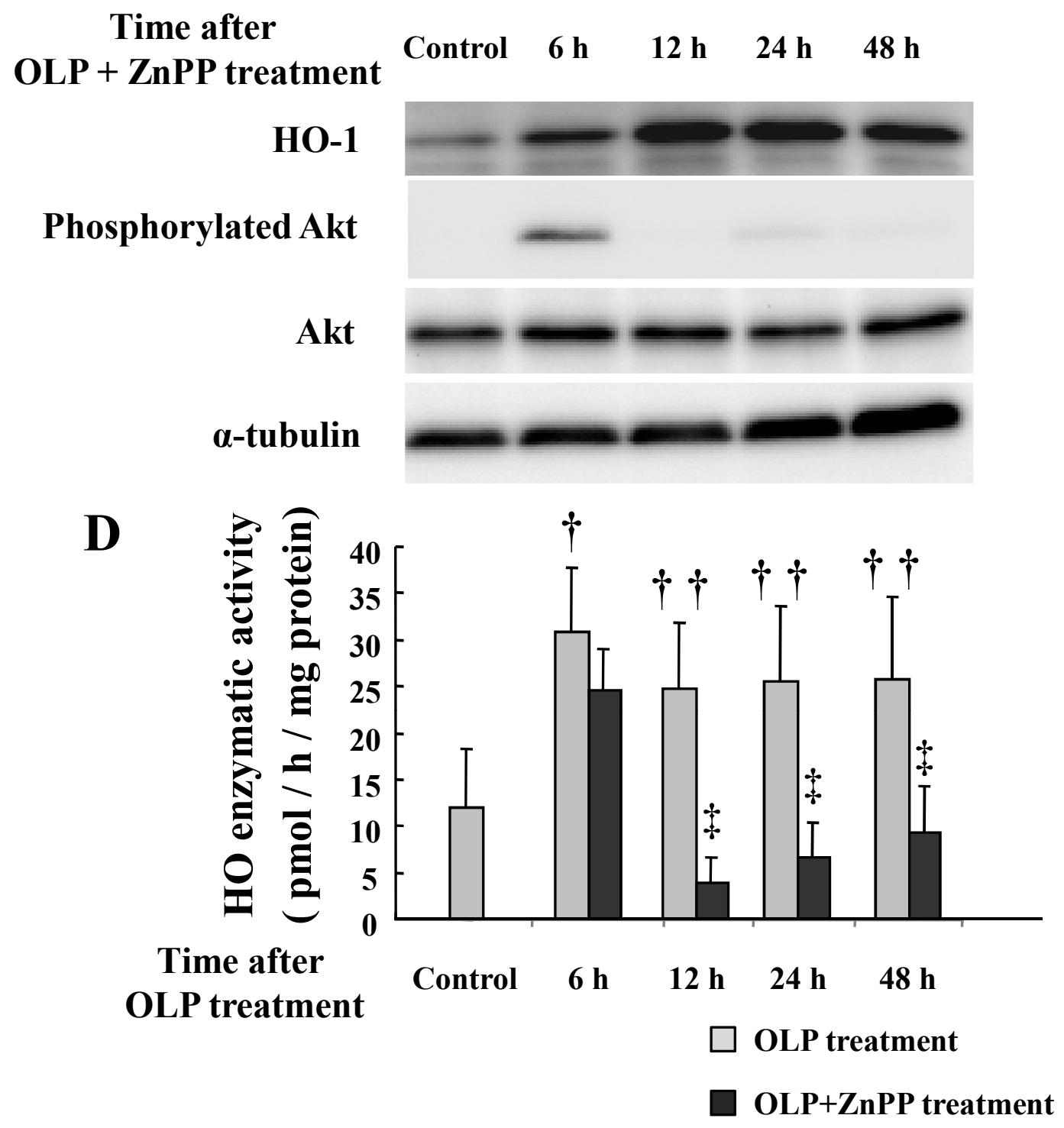

Control

CoPP-treated rat

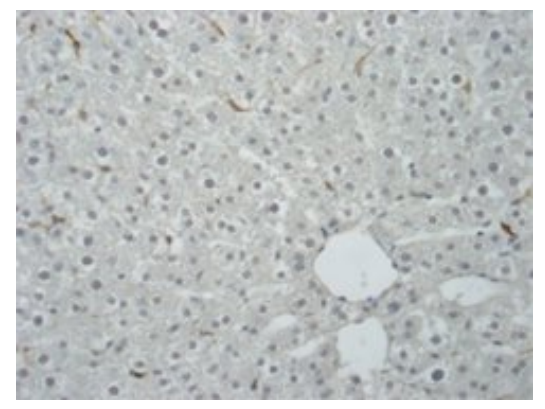

$\mathbf{F}$

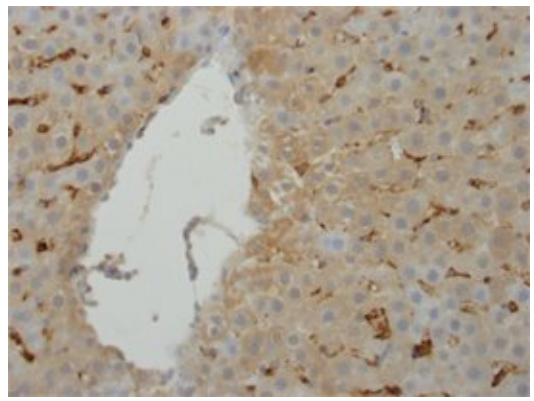

OLP-treated rat

G

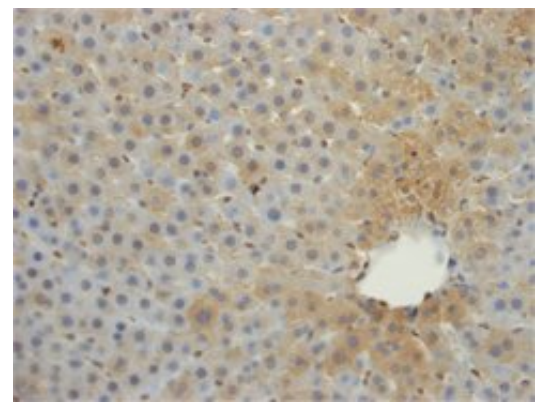




\section{Figure 4}

HE
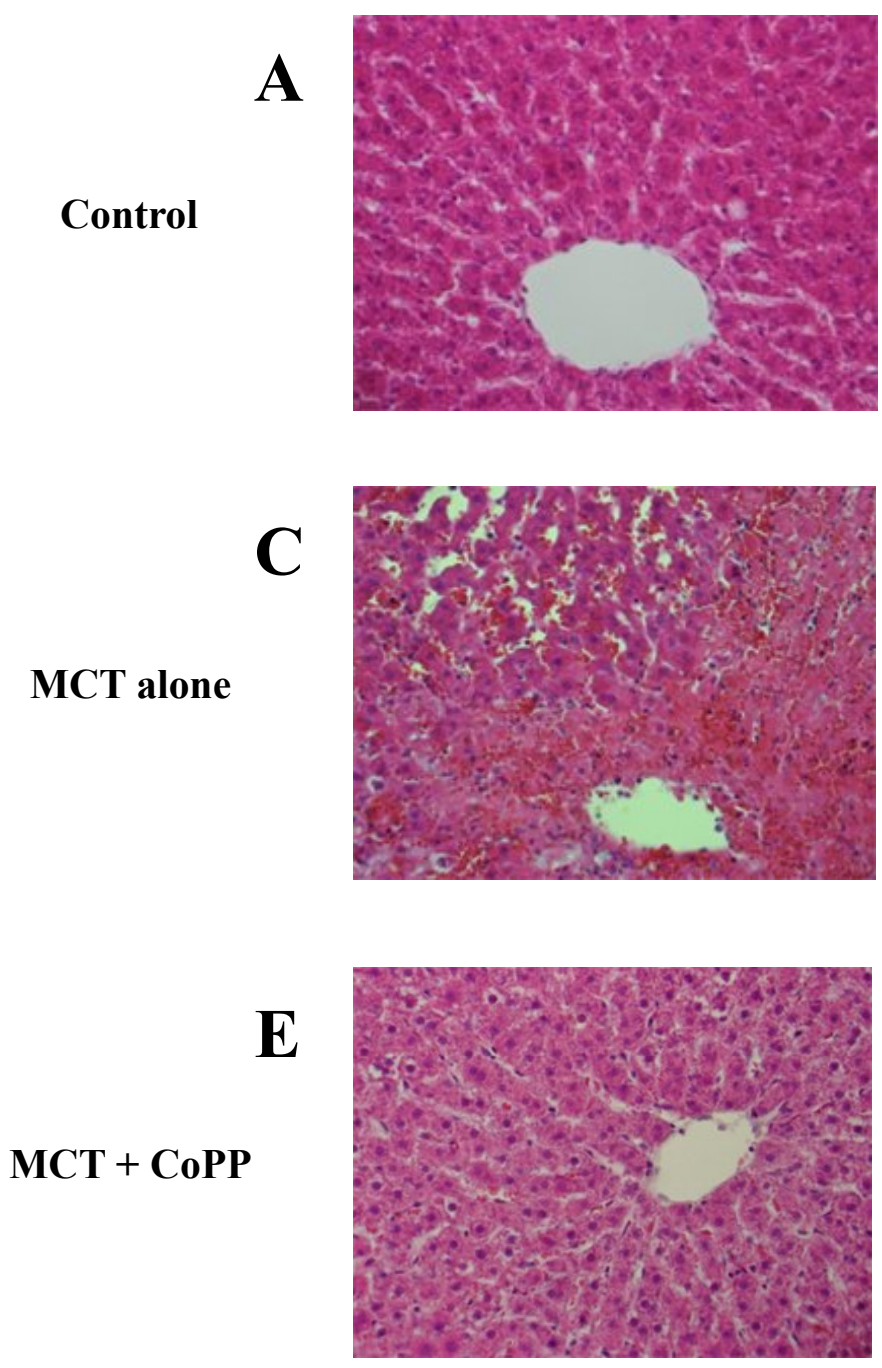

$\mathrm{MCT}+\mathrm{CoPP}+\mathrm{ZnPP}$
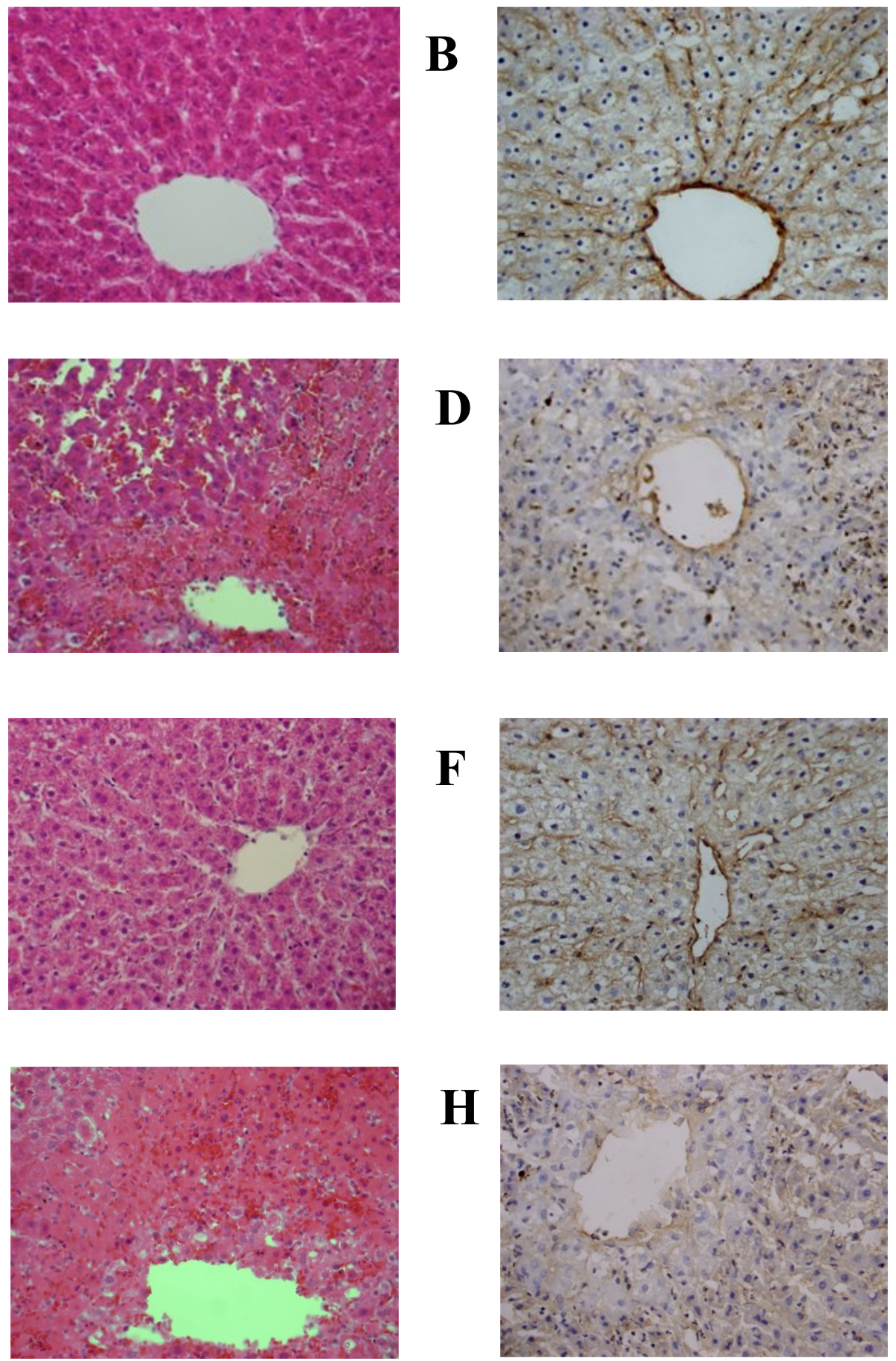

H

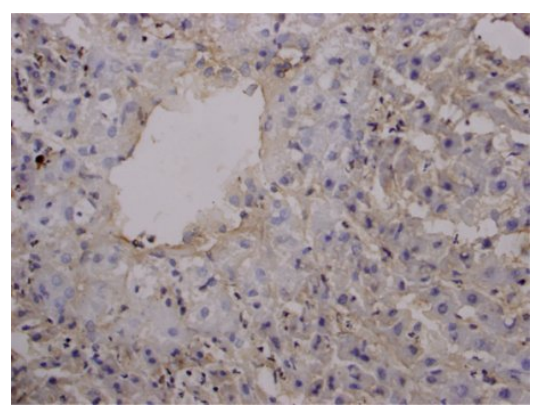




\section{Figure 5}

A

\section{HO-1}

Phosphorylated Akt

Akt

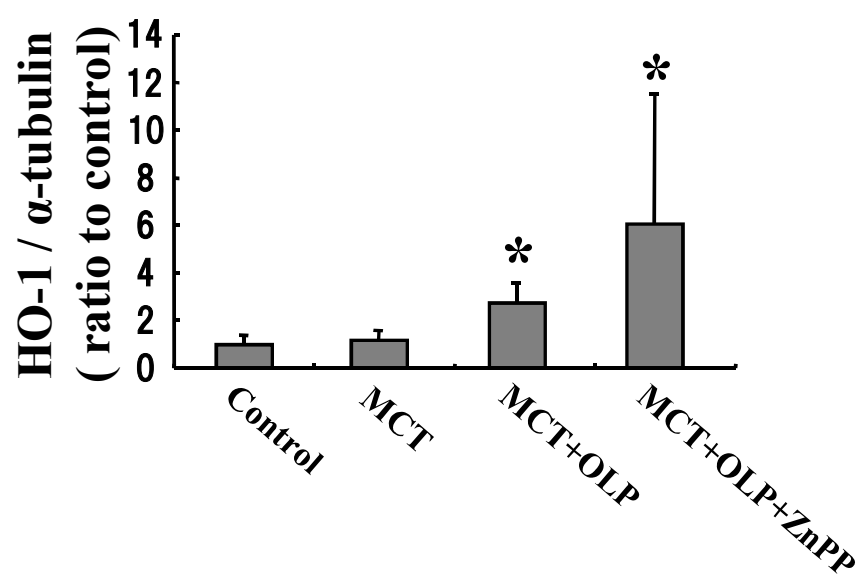

B

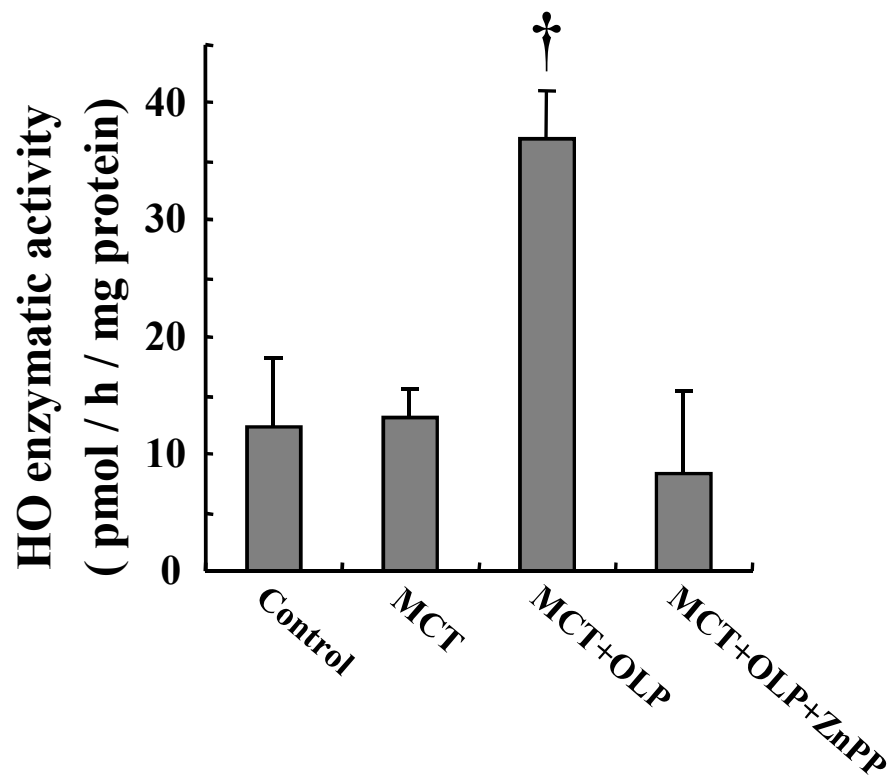




\section{Figure 6}

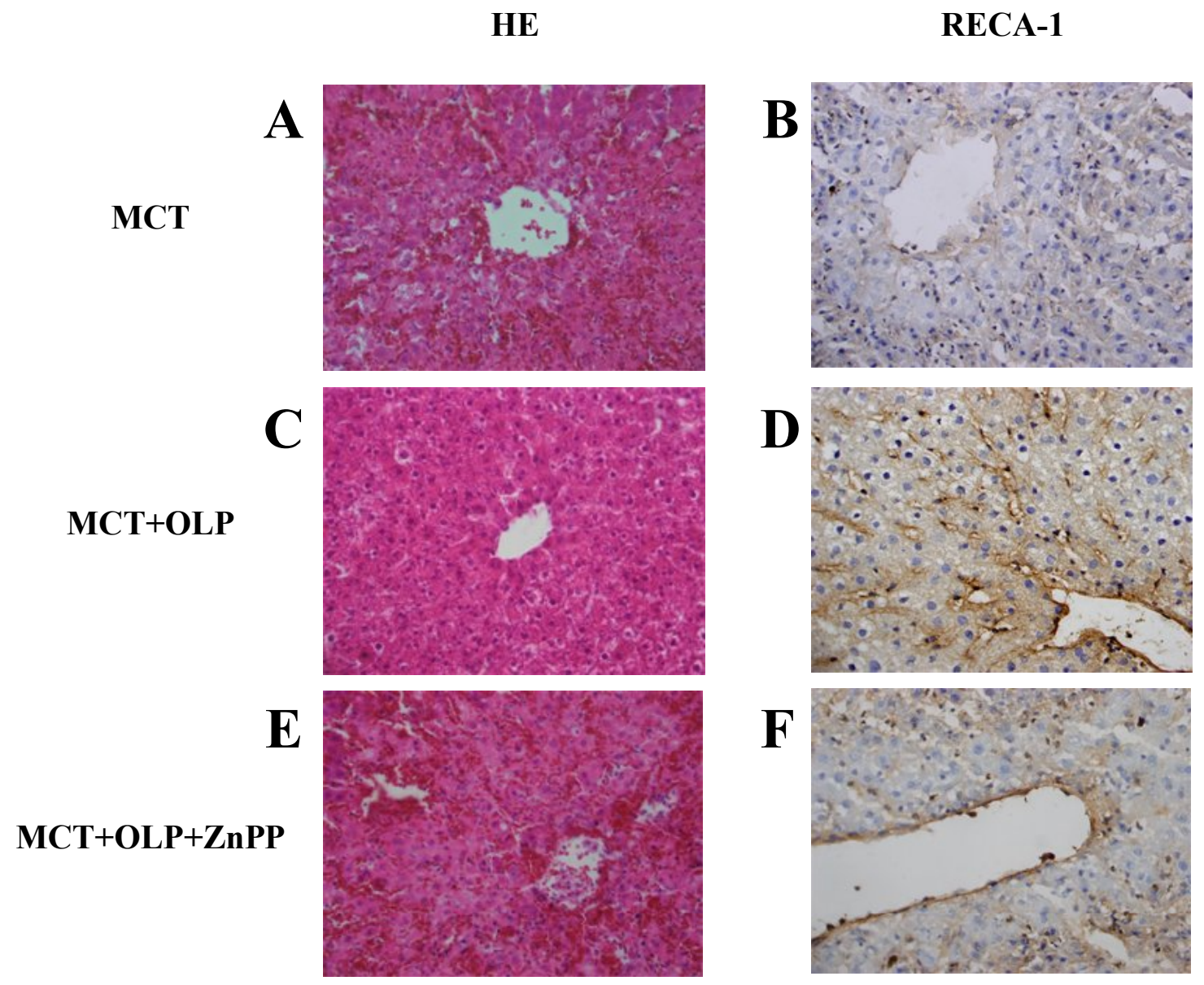




\section{Figure 7}

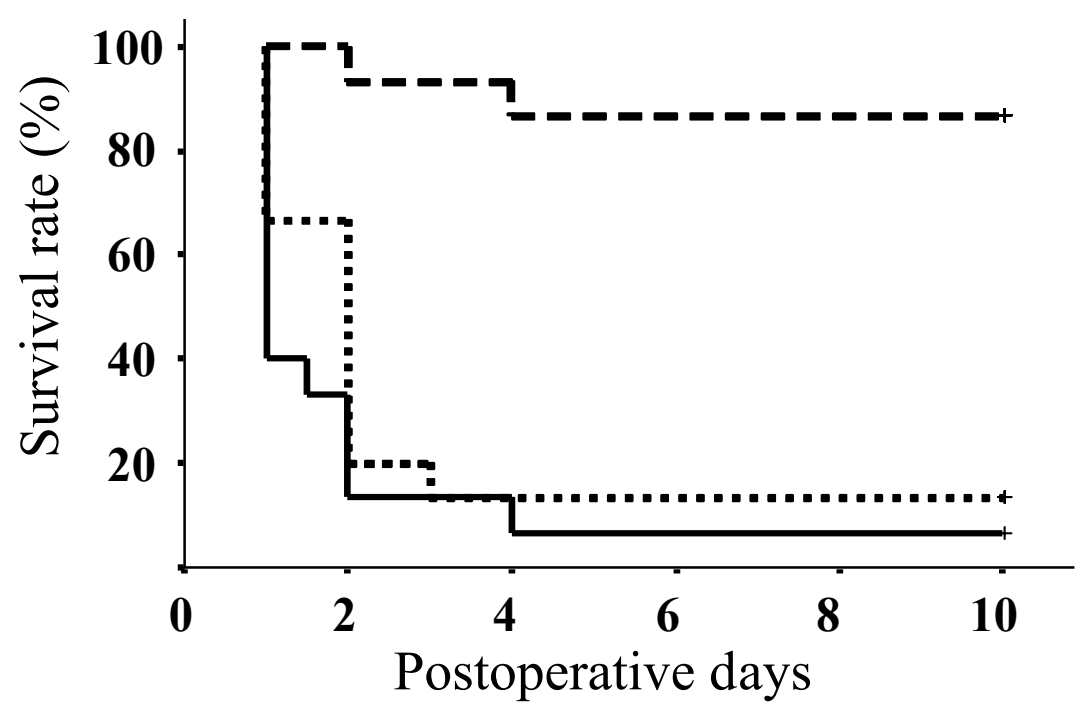




\section{Mini-Abstract}

Sinusoidal obstruction syndrome (SOS) induced by chemotherapy is of concern in hepatectomy for patients with hepatic colorectal metastases. We succeeded in inhibiting the occurrence of SOS in a rat model through induction of heme oxygenase-1 by pretreatment with olprinone, a phosphodiesterase III inhibitor. 


\section{Structured Abstract}

Objective: The aim of study was to investigate pharmacological treatment for sinusoidal obstruction syndrome (SOS).

Background: SOS is associated with oxaliplatin-based chemotherapy in patients with hepatic colorectal metastases. Patients with SOS have increased postoperative morbidity after major hepatectomy, but a method for effective prevention of SOS has not been established.

Methods: Male Sprague-Dawley rats were treated with cobalt protoporphyrin IX (CoPP) or olprinone (OLP), a phosphodiesterase III inhibitor, and hepatic HO-1 expression and HO enzymatic activity were determined. Monocrotaline (MCT) was given to rats to induce SOS, and blockage of SOS by CoPP or OLP-induced hepatic HO-1 was examined in these rats. Zinc protoporphyrin IX (ZnPP), a competitive HO inhibitor, was given to MCT-treated rats together with OLP to clarify the mechanism of protection against SOS. We also examined if OLP preserved remnant liver function after 70\% hepatectomy in MCT-treated rats.

Results: OLP upregulated hepatic HO-1 protein expression and $\mathrm{HO}$ enzymatic activity, and activated Akt protein. Administration of $\mathrm{ZnPP}$ to OLP-treated rats resulted in inhibition of $\mathrm{HO}$ activity and inactivation of Akt. Induction of $\mathrm{HO}-1$ by pretreatment with CoPP led to amelioration of SOS in histological findings and blockage of elevation of serum liver enzymes. Pretreatment with OLP gave a similar result and preserved remnant liver function, as indicated by improved survival after hepatectomy. ZnPP completely abolished the protective effects of OLP.

Conclusions: Protection of the liver from drug-induced injury by prior upregulation of HO-1 using OLP may have potential as a therapeutic strategy for prevention of SOS. 


\section{Financial disclosure}

This study entitled "Heme oxygenase-1 induced by a phosphodiesterase III inhibitor protects rat liver from sinusoidal obstruction syndrome" was supported in part by grants from the Scientific Research Fund of the Ministry of Education of Japan and from the Public Trust Surgery Research Fund. However, none of the authors has received funding from any organization with a real or potential interest in the subject matter, materials, equipment or devices discussed. 